\title{
CONTROLE TOLERANTE A FALHAS BASEADO EM ATUADORES VIRTUAIS COM HORIZONTE MÓVEL
}

\author{
T. V. da $\operatorname{COSTA}^{1}$, L. C. OLIVEIRA-LOPES ${ }^{2}$, A. M. F. FILETI ${ }^{1}$, F. V. da Silva ${ }^{1}$ \\ ${ }^{1}$ Universidade Estadual de Campinas, Faculdade de Engenharia Química \\ ${ }^{2}$ Universidade Federal de Uberlândia, Faculdade de Engenharia Química \\ E-mail para contato: flavio@feq.unicamp.br
}

\begin{abstract}
RESUMO - Os métodos para o controle tolerante a falhas complementam as malhas de controle de uma planta industrial utilizando dispositivos capazes de lidar com os eventos anormais de operação, evitando que falhas no processo comprometam sua operabilidade. Neste trabalho, as considerações sobre o mau funcionamento de atuadores e o estudo de técnicas capazes de acomodar seus efeitos são apresentadas. Esse estudo fundamenta-se em estratégias de controle reconfigurável aplicadas a sistemas multivariáveis. Uma extensão recentemente proposta na literatura, dirigida ao uso de atuadores virtuais com horizonte móvel é discutida e explorada, para os quais são feitas as considerações sobre sua implementação e aplicabilidade. A técnica é demonstrada em um estudo de caso com falhas em suas variáveis de entrada no qual o esforço de controle das redundâncias funcionais é redirecionado para a contenção dos efeitos da falha e manutenção da estabilidade na estrutura de controle.
\end{abstract}

\section{INTRODUÇÃO}

Sistemas de controle tolerante a falhas em processos (Mhaskar et al. 2013) compreendem um conjunto de técnicas capazes de intervir na estrutura de controle de um sistema com mau funcionamento em seus componentes com a finalidade de evitar a perda de desempenho e estabilidade, permitindo que o processo continue operacional. A intervenção do controle tolerante nos efeitos indesejáveis da falha, considerando técnicas de controle reconfigurável, é associada à alteração da estrutura do controle nominal, por modificação da composição de entradas e saídas destinadas ao controle em malha fechada para que o processo seja adequado ao novo cenário imposto pela falha no sistema (Blanke et al., 2006).

Destacam-se dentre as técnicas baseadas no controle reconfigurável, aquelas fundamentadas no rastreamento do comportamento nominal do sistema, como o método baseado nos atuadores e sensores virtuais, apresentados por Steffen (2005), com contribuições recentes encontradas na literatura como em Nazari et al. (2013) e Rotondo et al. (2014). Suas principais características consistem no redirecionamento dos sinais de entrada e na reconstrução dos sinais das saídas da malha de controle, recuperando as características dinâmicas do processo em situação de falha de acordo com suas condições normais de operação. As propriedades do método baseado nos sensores e atuadores virtuais são, dentre outras, aquelas relacionados à garantia de estabilidade do sistema, a recuperação do seu desempenho e a manutenção da estratégia de controle nominal. 
A perda de estabilidade de um processo pode ser comum em sistemas em malha fechada, quando suas variáveis de entrada e saída são atingidas por uma falha. Além disso, soma-se o fato de que muitos processos químicos são instáveis em malha aberta. Assim, garantir a estabilidade de um processo sujeito a falhas é característica essencial de uma estratégia de controle tolerante, pois conserva sua funcionalidade até que medidas de mitigação sejam efetuadas, impedindo paradas inoportunas de plantas em processos industriais.

Com a estabilidade garantida, procede-se com a retomada do desempenho. Entende-se que a perda de desempenho deve ser acompanhada e minimizada quando possível, considerando a extensão da falha no sistema. Deste modo, o sistema sujeito a falhas pode operar em conjunto com o controle tolerante até que sua manutenção seja viável. Além disso, o controle tolerante deve ser pouco intrusivo, como discutido em Richter et al. (2007). Nesse sentido, a manutenção da estratégia de controle nominal é relevante, principalmente em processos químicos, quando consideradas as diferentes estratégias de controle que podem ser encontradas em suas malhas. No método dos atuadores e sensores virtuais, essa tarefa é realizada por um mecanismo chamado ocultamento de falhas (Steffen, 2005), procedimento que simula o comportamento nominal da planta, enquanto a sua reestruturação é efetuada.

Considerando o exposto, este trabalho apresenta o método para controle tolerante em malhas com falhas em suas variáveis de entrada, a partir de técnicas fundamentadas nos atuadores virtuais. Nesse contexto, a reconfiguração estrutural do sistema é realizada a partir de técnicas de otimização dinâmica, em que se considera o desvio da planta em relação a sua condição nominal em um horizonte futuro. Assim, a consideração sistemática da retomada de desempenho é efetuada por uma camada externa de otimização contemplando esse desvio em regime permanente. A técnica é aplicada a estruturas de controle em processos químicos para sistemas multivariáveis.

\section{FUNDAMENTAÇÃO TEÓRICA}

Neste trabalho, o comportamento dinâmico do processo é representado por modelos lineares discretos e invariantes no tempo sob condições normais de operação e na presença de falhas para os estados $x_{m}$ e $x_{p}$, respectivamente (Equações 1 e 2):

$$
\begin{aligned}
& x_{m}(k+1)=A_{m} x_{m}(k)+B_{m} u_{m}(k), y_{m}(k)=C x_{m}(k) \\
& x_{p}(k+1)=A_{p} x_{p}(k)+B_{p} u_{p}(k), y_{p}(k)=C x_{p}(k)
\end{aligned}
$$

O método de reconfiguração baseado no atuador virtual utiliza uma estratégia que regula o desvio representado pelo estado $x_{\Delta}=x_{p}-x_{m}$, compensando a diferença entre os sistemas, descrito por alterações na estrutura da matriz de entradas $\left(A_{m}=A_{p}, B_{m} \neq B_{p}\right)$ para situações de falha nos atuadores. O procedimento é representado no sistema exposto na Equação 3 (Richter, 2011), adaptado para uma discussão no domínio discreto. 
atuador virtual: $\left\{\begin{array}{l}x_{\Delta}(k+1)=A_{\Delta} x_{\Delta}(k)+B_{\Delta} u_{p}(k), x_{\Delta}(0)=0 \\ u_{p}=M x_{\Delta}(k)+N u_{m}(k) \\ y_{m}=y_{p}+C x_{\Delta}(k) \\ A_{\Delta} \triangleq A-B_{p} M, B_{\Delta} \triangleq B-B_{p} N\end{array}\right.$

De acordo com esta definição, o problema consiste em projetar a matriz de realimentação $M$ e a matriz do termo de avanço $N$ para que o desvio $x_{\Delta}$ seja regulado para sua origem, igualando o comportamento da planta com falhas ao seu comportamento nominal, caso existam graus de liberdade suficientes para a tarefa de reconfiguração no sistema.

De maneira similar, pode-se considerar o método do ponto de vista de um problema de otimização dinâmica, no qual o estado nominal $\left(x_{m}\right)$ é uma referência a ser seguida. Assim, uma função objetivo que considera o desvio $x_{\Delta}$ somado ao esforço de controle destinado para a tarefa de reconfiguração é estabelecida (Costa et al., 2013). Neste trabalho, emprega-se uma um problema de otimização com horizonte infinito e $N_{v}$ graus de liberdade como apresentado na Equação 4. Assim, o problema de reconfiguração é tratado de forma análoga aos métodos apresentados na literatura para o controle preditivo (Rawlings e Mayne, 2009).

$$
\begin{aligned}
\min _{x_{\delta}, u_{\delta}} J & =x_{\delta}^{T}(k+N \mid k) Q_{\infty} x_{\delta}(k+N \mid k)+\sum_{i=1}^{N v}\left\{\left\|x_{\delta}(k+i-1 \mid k)\right\|_{Q_{\Delta}}^{2}+\left\|u_{\delta}(k+i-1 \mid k)\right\|_{R_{\Delta}}^{2}\right\} \\
\text { s.a } & x_{\delta}=x_{\Delta}-\bar{x}_{\Delta} \text { e } u_{\delta}=u_{p}-\bar{u}_{p} \\
& x_{\Delta}(k+i)=A x_{\Delta}(k+i-1)+B_{m} u_{m}(k+i-1)-B_{p} u_{p}(k+i-1) \\
& u_{p} \in U
\end{aligned}
$$

Na função objetivo, o espaço $U$ é definido por restrições lineares. As variáveis de decisão $x_{\delta} \in \mathfrak{R}^{N_{v} \cdot n_{x}}$ e $u_{\delta} \in \mathfrak{R}^{N_{v} \cdot n_{u}}$, são perturbações do desvio $\left(x_{\Delta}\right)$ e do sinal de controle $\left(u_{p}\right)$, ponderadas por matrizes positivo definidas $Q_{\Delta}$ e $R_{\Delta}$, respectivamente. Por hipótese, assume-se o valor do controle nominal $u_{m}$ constante no horizonte de predição $N_{v}$. Além disso, os sinais de controle $u_{m}$ e $u_{p}$ em relação aos seus estados estacionários são considerados nulos na parcela de custo terminal (primeira parcela da função objetivo). A matriz $Q_{\infty}$, presente no custo terminal, é encontrada para sistemas estáveis a partir da solução da equação de Lyapunov, de acordo com a Equação 5 (Maciejowski, 2001):

$$
A^{T} Q_{\infty} A-Q_{\infty}=-Q_{\Delta}
$$

As variáveis de decisão são calculadas em relação a um conjunto de valores admissíveis ou de referência: $\bar{x}_{\Delta}$ e $\bar{u}_{p}$. Deste modo, as considerações sobre as redundâncias disponíveis no sistema são realizadas de forma explícita no problema de reconfiguração. Estes valores de referência são obtidos em duas etapas. Na primeira etapa, os estados e as entradas $\left(\bar{x}_{m}, \bar{u}_{m}\right)$ em estado estacionário são calculados em relação ao estado nominal, para uma referência imposta, de forma que: $y_{m} \rightarrow w$ (Equação 6). Considera-se que o controlador é capaz de atingir a referência para um cenário livre de falhas. 


$$
\left[\begin{array}{cc}
I-A & -B_{m} \\
C & 0
\end{array}\right]\left[\begin{array}{l}
\bar{x}_{m} \\
\bar{u}_{m}
\end{array}\right]=\left[\begin{array}{l}
0 \\
w
\end{array}\right]
$$

$\mathrm{Na}$ etapa seguinte, a diferença entre o comportamento nominal e o comportamento da planta com falha em regime permanente é calculada em uma camada externa de otimização (Equação 7). Sendo $Q_{\mathrm{s}}$ uma matriz positivo definida que pondera a diferença entre os estados. Assim, são obtidos os valores de referência $\left(\bar{x}_{\Delta}, \bar{u}_{p}\right)$, que devem ser seguidos pelo problema de rastreamento dinâmico definido na Equação 4.

$$
\begin{gathered}
\min _{\bar{x}_{p}, \bar{u}_{p}} J=\left(\bar{x}_{m}-\bar{x}_{p}\right)^{T} Q_{s}\left(\bar{x}_{m}-\bar{x}_{p}\right) \\
\text { s.a }(I-A) \bar{x}_{p}-B_{p} \bar{u}_{p}=0 \\
\quad \bar{u}_{p} \in U
\end{gathered}
$$

O desvio $\bar{x}_{\Delta}$ pode ser nulo para todos os estados, caso as redundâncias do sistema sejam suficientes para suprir a demanda ocasionada pela falha. Assim, existe a reconfiguração completa do sistema que retoma seu comportamento nominal a partir de suas redundâncias. No entanto, pode ser impossível atingir um valor nulo para todos os elementos de $\bar{x}_{\Delta}$, caso em que um desvio permanente em relação a certos estados do sistema sujeito a falhas deve ser tolerado.

\section{ESTUDO DE CASO}

Nesta seção o problema de controle aplicado a um processo multivariável de neutralização (Hall e Seborg, 1989) é apresentado como exemplo ilustrativo para o método. O sistema, cujo modelo linear é descrito em Conner e Seborg (2005), consiste em dois tanques para neutralização de correntes com ácidos e bases fortes. As simulações foram realizadas no software Scilab 5.5.0.

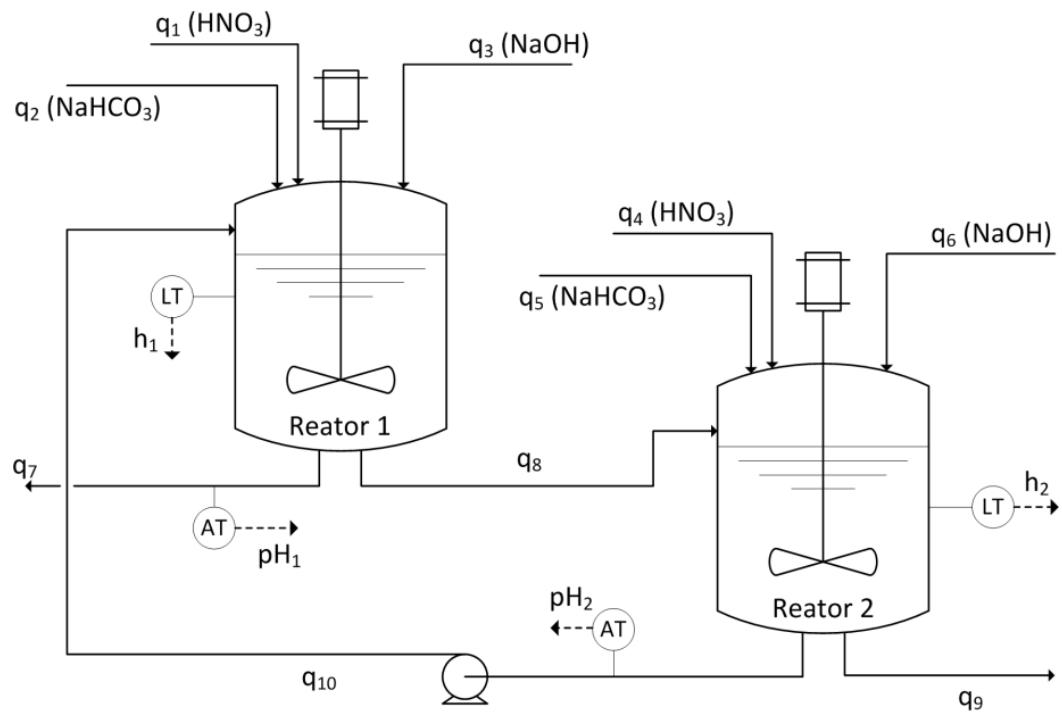

Figura 1- Diagrama esquemático do processo. 
As correntes de entrada são constituídas de ácido nítrico $\left(\mathrm{HNO}_{3}, q_{1}\right.$ e $\left.q_{4}\right)$, hidróxido de sódio $\left(\mathrm{NaOH}, q_{3}\right.$ e $\left.q_{6}\right)$ e bicarbonato de sódio $\left(\mathrm{NaHCO}_{3}, q_{2}\right.$ e $\left.q_{5}\right)$. O efluente do primeiro reator é divido e alimentado ao segundo reator $\left(q_{8}\right)$ sendo neutralizado em conjunto com as demais correntes de entrada deste reator. Uma corrente de reciclo $\left(q_{10}\right)$ é utilizada para realimentar o primeiro reator enquanto as correntes $q_{7} \mathrm{e} q_{9}$ deixam o processo. O conjunto total de entradas e as saídas do sistema são definidas por $y=\left[\begin{array}{llll}h_{1} & p H_{1} & h_{2} & p H_{2}\end{array}\right]^{T}$ e $u=\left[\begin{array}{llll}q_{1} & q_{3} & q_{4} & q_{6}\end{array}\right]^{T}$.

As malhas de controle nominal consistem no controle do nível e $\mathrm{pH}$ no segundo reator, com o seguinte pareamento: malha $1\left(h_{2}-q_{4}\right)$ e malha $2\left(p H_{2}-q_{6}\right)$. As malhas são compostas por dois controladores PI (proporcionais e integrais) com constantes: $k_{c l}=1 ; k_{c 2}=4 ; \tau_{1}=0,6 \mathrm{e}$ $\tau_{2}=0,6$ em unidades compatíveis. O primeiro reator opera em malha aberta. Neste caso, as correntes de entrada $\left(q_{1}\right.$ e $\left.q_{3}\right)$ podem ser utilizadas como redundâncias funcionais para auxiliar na recuperação do sistema em caso de falhas nos atuadores. Considera-se que todas as variáveis passíveis de manipulação possuam limites físicos na faixa $[-4,4]$ em relação ao estado estacionário. As variáveis $q_{2}$ e $q_{5}$ são mantidas constantes.

A simulação em condições normais de operação para o sistema discretizado com um tempo de amostragem $\left(T_{s}\right)$ igual a 2 segundos é apresentada na Figura 2, para as variáveis desvio partindo do estado estacionário. A simulação em malha fechada teve como objetivo manter o nível do segundo reator constante enquanto uma perturbação degrau na malha de controle de $\mathrm{pH}$ com amplitude igual à 1 em relação ao seu estado estacionário foi aplicada. Nota-se, aqui, o acoplamento das malhas, já que a alteração de $\mathrm{pH}$ no reator implica no aumento da vazão de base $\left(q_{6}\right)$ que, consequentemente, demanda uma redução da vazão de ácido $\left(q_{4}\right)$ para manutenção do nível na referência.
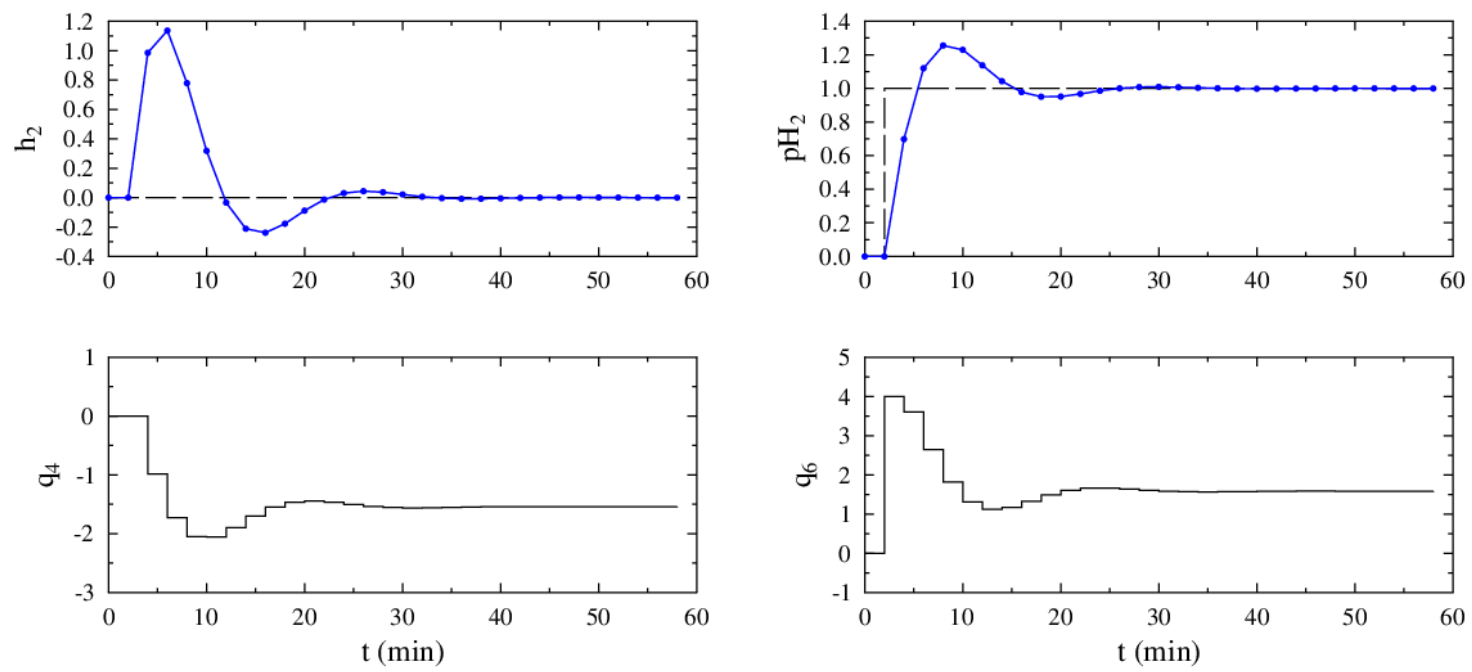

Figura 2 - Comportamento dinâmico nominal do sistema de neutralização em malha fechada.

A situação de falha é ilustrada na Figura 3. Considera-se uma falha no atuador da malha de controle de $\mathrm{pH}$ no segundo reator. A falha simulada consiste no bloqueio do atuador $\left(q_{6}\right)$ instantes após a mudança na referência do $\mathrm{pH}$. O sistema, com o bloqueio do atuador, começa então a operar em malha aberta e retorna ao seu ponto de operação inicial. A falha não causa instabilidade no sistema já que este é estável em malha aberta, no entanto, impede o sistema de cumprir seus objetivos de controle ocasionando perda em seu desempenho. 
A reconfiguração da estrutura de controle para o cenário de falha no atuador da segunda malha de controle utiliza a corrente de base a montante do primeiro reator $\left(q_{3}\right)$ para suprir a demanda do sistema para a alteração desejada no valor de $\mathrm{pH}$ da mistura no segundo reator $\left(\mathrm{pH}_{2}\right)$.
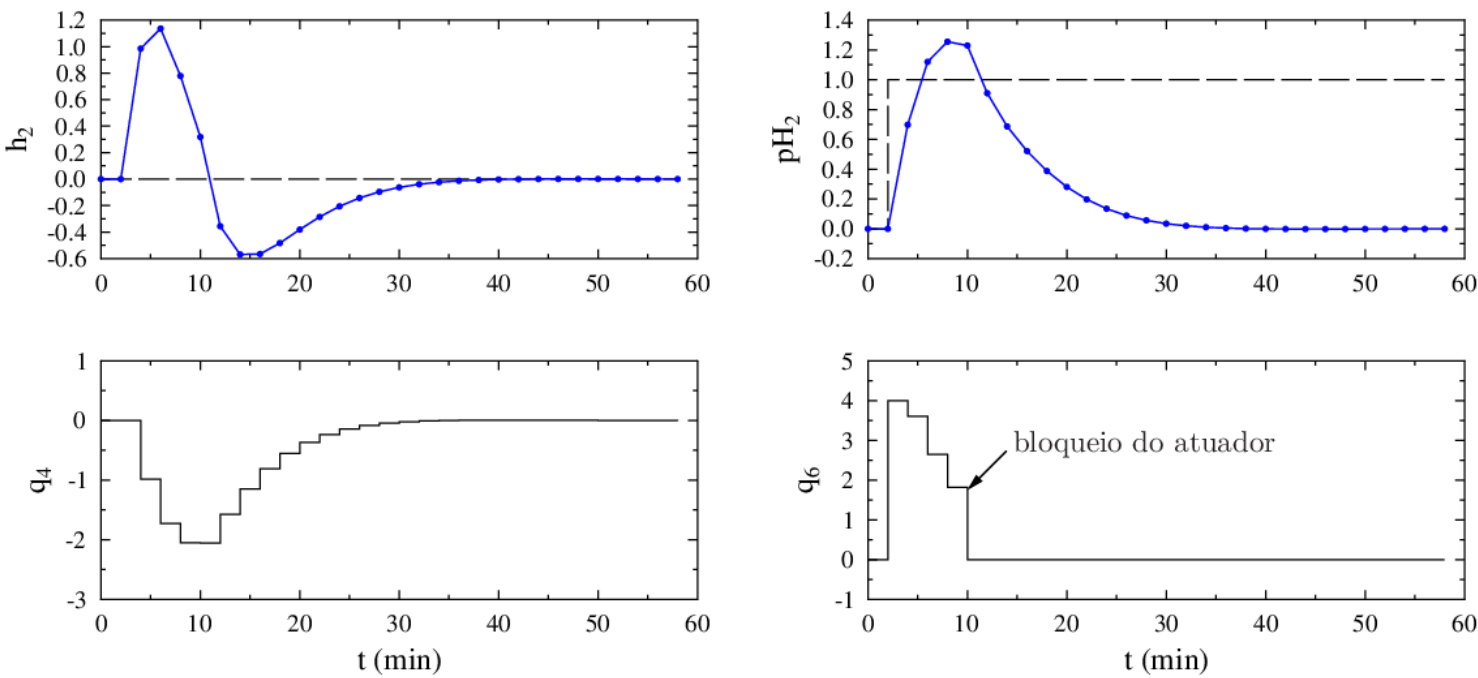

Figura 3 - Comportamento dinâmico do sistema com falha no atuador da linha de base $\left(\mathrm{q}_{6}\right)$.

Os problemas de otimização utilizados no método de reconfiguração foram resolvidos pela rotina de programação quadrática quapro. O procedimento de regulação do estado que representa o desvio da planta em relação ao seu comportamento nominal é apresentado no plano de fases para as variáveis de decisão $x_{\delta}$ e $u_{\delta}$, na Figura 4. Pode-se observar que o sistema reconfigurado é conduzido assintoticamente para a origem, estabelecendo a acomodação da falha pela reestruturação da malha de controle nominal com o uso da redundância $\left(q_{3}\right)$.
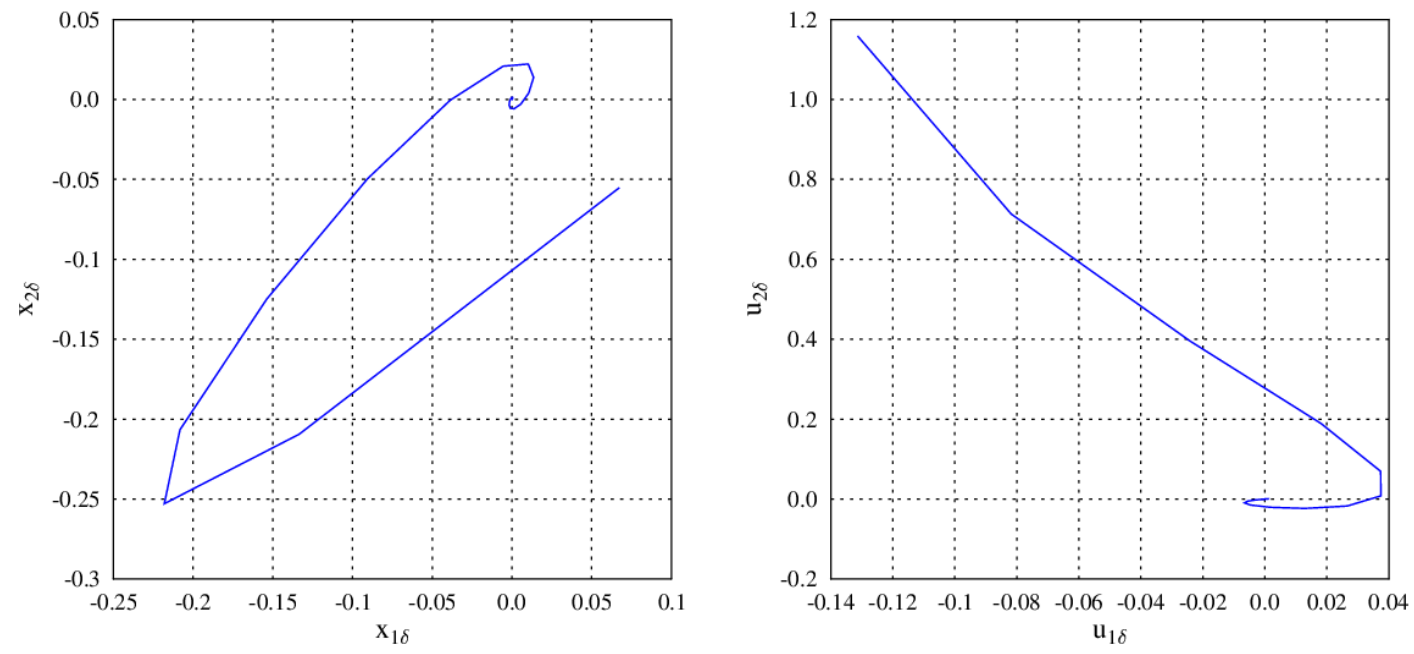

Figura 4 - Evolução no plano de fases para as variáveis de decisão $x_{\delta}$ e $u_{\delta}$

O comportamento temporal do sistema reconfigurado é apresentado na Figura 5. No gráfico, é possível observar o sinal de falseamento das variáveis controladas $\left(y_{m}\right)$, que é simulado pela estratégia de controle reconfigurável com o objetivo de omitir a falha para o 
controlador nominal. Os sinais de controle $u_{m}$ são modificados em linha, pois o controlador nominal não é comutado, e são alterados para as trajetórias de controle $\left(u_{p}\right)$ que utiliza as variáveis funcionais $q_{3}$ e $q_{4}$.

A falha é mitigada pela transição de sinais sem que as restrições físicas das variáveis de entrada fossem violadas. Após um período de transição, o sinal nominal da planta é alcançado, igualando o comportamento do sistema com falhas ao seu comportamento nominal. Isto ocorre, pois apesar da perda de um atuador, o processo continua controlável a partir das demais variáveis de entrada, sendo possível efetuar a reconfiguração completa com a estratégia do atuador virtual.
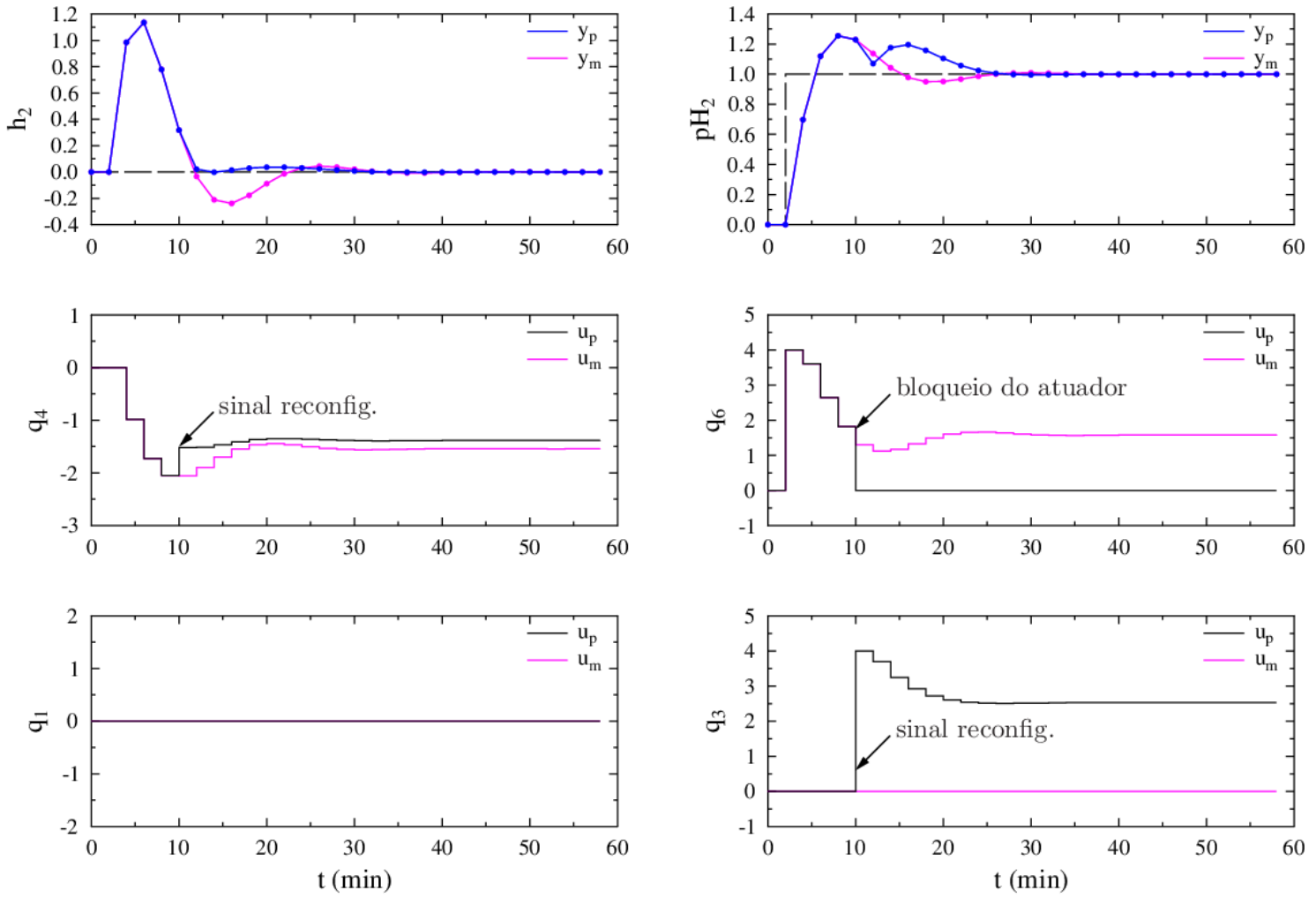

Figura 5 - Comportamento dinâmico do sistema em situação de falha na presença da estratégia de controle tolerante.

\section{CONCLUSÕES}

Neste trabalho, foi considerada a técnica de controle tolerante baseada no atuador virtual com ocultamento da falha para o sistema de controle nominal. Um procedimento de otimização com horizonte móvel foi utilizado para o rastreamento do comportamento nominal da planta a partir de sinais de referência considerando o desvio admitido e o sinal de controle destinado às variáveis funcionais do sistema. Os resultados apresentados para o estudo de caso de um sistema de neutralização multivariável demonstraram que a técnica proposta é capaz de redirecionar os sinais de controle, efetuando a reestruturação do sistema e contornando, assim, os efeitos da falha causada pelo bloqueio da variável de entrada de uma das malhas de controle. 
Embora, não se tenha abordado nesse estudo, o máximo desvio da planta sujeita a falhas em relação ao seu estado nominal pode ser considerado na definição da função objetivo. Este procedimento, no entanto, pode causar problemas de não viabilidade do procedimento de otimização. Nesse caso, pode ser necessário do uso de variáveis folga em conjunto com técnicas de controle robusto. Este problema, em conjunto com a consideração de discrepâncias entre planta e modelo e perturbações para sistemas reais são temas para futuros estudos a serem apresentados no contex to deste trabalho.

\section{REFERÊNCIAS}

BLANKE, M.; KINNAERT, M.; LUNZE, J.; STAROSWIECKI, M. Diagnosis and FaultTolerant Control. Berlin: Springer, 2006.

CONNER, S.; SEBORG, D. Assessing the need for process re-identification. Industrial \& Engineering chemistry research, v. 44, n. 8, p. 2767-2775, 2005.

COSTA, T. V.; FILETI, A. M. F.; OLIVEIRA-LOPES, L. C.; SILVA, F. V. Control reconfiguration of chemical processes subjected to actuator faults: A moving horizon approach. The 14th International Symposium on Intelligent Systems and Control. Marina del Rey: 2013. p. 377-384.

HALL, R.; SEBORG, D. Modelling and Self-Tuning Control of a Multivariable $\mathrm{pH}$ Neutralization Process Part I: Modelling and Multiloop Control. American Control Conference, 1989. p. 1822-1827.

MACIEJOWSKI, J. Predictive control with constraints. London: Prentice Hall, 2001.

MHASKAR, P.; LIU, J.; CHRISTOFIDES, P. Fault-Tolerant Process Control. London: Springer, 2013.

NAZARI, R.; SERON, M.; DONÁ, J. Fault-tolerant control of systems with convex polytopic linear parameter varying model uncertainty using virtual sensor based controller reconfiguration. Annual Reviews in Control, v. 37, p. 146-153, 2013.

RAWLINGS, J.; MAYNE, Q. Model predictive control: Theory and design. Madison: Nob Hill Pub., 2009.

RICHTER, J. Reconfigurable Control of Nonlinear Dynamical Systems. Springer, 2011.

RICHTER, J. H.; SCHLAGE, T.; LUNZE, J. Control reconfiguration of a thermofluid process by means of a virtual actuator. Control Theory \& Applications, n. 6, p. 1606-1620, 2007.

ROTONDO, D.; NEJJARI, F.; PUIG, V. A virtual actuator and sensor approach for fault tolerant control of LPV systems. Journal of Process Control, v. 24, n. 3, p. 203-222, 2014.

STEFFEN, T. Control Reconfiguration of Dynamical Systems: Linear Approaches and Structural Tests. Heidelberg: Springer, 2005. 